\title{
Chromosome-level genome of Poropuntius huangchuchieni provides a diploid progenitor-like reference genome for the allotetraploid
}

\section{Cyprinus carpio}

\author{
Lin Chen ${ }^{1}$, Bijun $\mathrm{Li}^{1}$, Baohua Chen ${ }^{1}$, Chengyu Li ${ }^{1}$, Zhixiong Zhou ${ }^{1}$, Weidi Yang ${ }^{1}$, Tao \\ $\mathrm{Zhou}^{1}$, and Peng $\mathrm{Xu}^{1}$ \\ ${ }^{1}$ Fujian Key Laboratory of Genetics and Breeding of Marine Organisms
}

November 5, 2020

\begin{abstract}
The diploid Poropuntius huangchuchieni in the cyprinid family, which is widely distributed in the Mekong and Red River basins, is one of the most closely related diploid progenitor-like species of allotetraploid common carp, which was generated by merging of two diploid genomes during evolution. Therefore, the P. huangchuchieni genome is essential for polyploidy evolution studies in Cyprinidae. Here, we report a high-quality chromosome-level genome assembly of P. huangchuchieni by integrating Oxford Nanopore and Hi-C technology. The assembled genome size was $1021.38 \mathrm{Mb}, 895.66 \mathrm{Mb}$ of which was anchored onto 25 chromosomes with a N50 of $32.93 \mathrm{Mb}$. The genome contained $486.28 \mathrm{Mb}$ repetitive elements and 24,099 protein-coding genes. Approximately $95.9 \%$ of the complete BUSCOs were detected, suggesting a high completeness of the genome. Evolutionary analysis revealed that P. huangchuchieni diverged from Cyprinus carpio at approximately 12 Mya. Genome comparison between P. huangchuchieni and the B subgenome of C. carpio provided insights into chromosomal rearrangements during the allotetraploid speciation. With the complete gene set, 17,474 orthologous genes were identified between P. huangchuchieni and C. carpio, providing a broad view of the gene component in the allotetraploid genome, which is critical for future genetic analyses. The high-quality genomic dataset created for P. huangchuchieni provides a diploid progenitor-like reference for the evolution and adaptation of allotetraploid carps.
\end{abstract}

\section{Hosted file}

MER-Chromosome-level genome assembly of Poropuntius huangchuchieni-10-17.pdf available at https://authorea.com/users/373343/articles/491040-chromosome-level-genome-ofporopuntius-huangchuchieni-provides-a-diploid-progenitor-like-reference-genome-for-theallotetraploid-cyprinus-carpio 